\title{
Sequential Sampling Strategy for the Modeling of Parameterized Microwave and RF Components
}

\author{
Dirk Deschrijver \\ Dept. of Information Techn. \\ Ghent University - IBBT \\ 9000 Ghent, Belgium \\ dirk.deschrijver@intec.ugent.be karel.crombecq@intec.ugent.be \\ Karel Crombecq \\ Dept. of Information Techn. \\ Ghent University - IBBT \\ 9000 Ghent, Belgium
}

\author{
Huu Minh Nguyen \\ Dept. of Information Techn. \\ Ghent University - IBBT \\ 9000 Ghent, Belgium \\ hnguyen@intec.ugent.be
}

\author{
Tom Dhaene \\ Dept. of Information Techn. \\ Ghent University - IBBT \\ 9000 Ghent, Belgium \\ tom.dhaene@intec.ugent.be
}

\begin{abstract}
Accurate modeling of parameterized microwave and RF components often requires a large number of full-wave electromagnetic simulations. In order to reduce the overall simulation cost, a sequential sampling algorithm is proposed that selects a sparse set of data samples which characterize the overall response of the system. The resulting data samples can be fed into existing modeling techniques. The effectiveness of the approach is illustrated by a parameterized $\mathrm{H}$-shaped microwave antenna.
\end{abstract}

\section{INTRODUCTION}

Accurate circuit models are needed for the design, study and optimization of passive microwave and RF components [1][7]. To this end, parameterized analytical models are needed which characterize the response of the system as a function of frequency and layout variables. Such models are fast to evaluate, and offer a lot of different analysis possibilities (e.g. optimization, sensitivity analysis and what-if analysis) [9].

The calculation of such models requires a large number of full-wave electromagnetic simulations, which is often time consuming [10]. In order to reduce the computational workload, a new sequential sampling strategy [11] is modified such that data samples are scattered in terms of all parameters, including angular frequency [12]. It automatically selects a suitable sample distribution, which accurately captures the dynamical behavior of the system. This set of representative data samples can be fed into an arbitrary modeling technique, provided that it can deal with scattered data. Since the selection of data samples is completely decoupled from the model building part, the new algorithm is much more flexible and generic, compared to existing sampling approaches [13]-[17].

The benefits of the sampling approach are illustrated by applying it to a scalable microwave $\mathrm{H}$-antenna example.

\section{PReliminaries And Notation}

Modeling algorithms are used to compute parametric models from S-parameter data $\{\vec{g}, H(\vec{g})\}$. These S-parameters $H(\vec{g}) \in \mathbb{C}$ depend on several parameters $\vec{g}=\left\{g^{(n)}\right\}_{n=1}^{N} \subset$ $\mathbb{R}^{N}$ such as the angular frequency and design variables (metallizations of a component, substrate parameters,...). To limit the overall cost of full-wave EM simulations, a sequential sampling strategy is proposed that determines up-front a sparse set of data samples that characterize the overall design space.

\section{Methodology of SAmpling Algorithm}

The sequential sampling algorithm starts from a small set of initial data samples, and selects additional data samples in a sequential way until the algorithm is terminated. It makes a trade-off between exploration and exploitation [18], [19] :

- Exploration is the act of exploring the design space in order to find key regions that have not yet been identified before. It does not involve the response of the system, but only the location of data samples over the design space. It ensures that data samples are spread as evenly as possible.

- Exploitation means that data samples are chosen in regions of the design space that are identified as potentially interesting. Regions where the response is highly dynamic require a finer sampling than regions with little variation.

For the exploration criterion, the density of data samples is quantified by computing a Voronoi tessellation of the data samples and by calculating the volume of each Voronoi cell (Sect IV). For the exploitation criterion, the dynamic variation of the reponse is quantified by computing simple local linear approximation models that are compared with the true system response (Sect V). Both criteria are combined to identify undersampled regions of the design space, and to determine the optimal location of additional data samples (Sect VI).

\section{EXPLORATION - VORONOI TESSELLATIONS}

The density of data samples is assessed by computing a Voronoi tessellation [20] of the design space and by estimating the volume of each cell. Cells having a large volume correspond to regions in the design space that are sampled sparsely. Assume that a discrete and pairwise distinct set of samples $P=\left\{\vec{p}_{k}\right\}_{k=1}^{K}$ in the design space is given. Then the Voronoi cell $C_{k}$ of $\vec{p}_{k}$ contains all samples in the design space lying closer to $\vec{p}_{k}$ than any other sample in $P$. The complete set of cells $\left\{C_{k}\right\}_{k=1}^{K}$ tessellates the design space, and is called the Voronoi tessellation corresponding to $P$. To compute the volume of each Voronoi cell, the unbounded cells near the border of the parameter ranges are bounded. Then, the volume (Vol) of each cell is estimated by Monte Carlo methods [21].

To assess the density of the data samples around $\vec{p}_{k}$, the following normalized metric $V\left(\vec{p}_{k}\right) \in[0,1]$ is introduced 


$$
V\left(\vec{p}_{k}\right)=\frac{\operatorname{Vol}\left(C_{k}\right)}{\operatorname{Vol}\left(C_{1}\right)+\ldots+\operatorname{Vol}\left(C_{K}\right)}
$$

Note that $V\left(\vec{p}_{k}\right)$ quantifies the portion of the design space that is contained within each Voronoi cell $C_{k}$ of $\vec{p}_{k}$.

\section{Exploitation - Local Linear ApProximations}

Regions of the design space with a high dynamical behavior are identified as follows. For each sample $\vec{p}_{k}$ in $P$, a suitable set of $V$ neighbouring data samples $N\left(\vec{p}_{k}\right)$ is chosen.

$$
N\left(\vec{p}_{k}\right)=\left\{\vec{p}_{k v}\right\}_{v=1}^{V} \text { with } N\left(\vec{p}_{k}\right) \subset P \backslash\left\{\vec{p}_{k}\right\}
$$

These neighbours are chosen in such a way that each direction of the design space is covered equally well. Using these neighbours, the gradient $\nabla H(\vec{g})$ is estimated from data [22]

$$
\nabla H(\vec{g})=\left(\frac{\partial H(\vec{g})}{\partial g^{(1)}}, \frac{\partial H(\vec{g})}{\partial g^{(2)}}, \ldots, \frac{\partial H(\vec{g})}{\partial g^{(N)}}\right)
$$

and leads to the best local linear approximation $\tilde{H}(\vec{g})$ at $\vec{p}_{k}$

$$
\tilde{H}(\vec{g})=H\left(\vec{p}_{k}\right)+\left.\nabla H(\vec{g})\right|_{p_{k}}\left(\vec{g}-\vec{p}_{k}\right)
$$

Note that $\left.\nabla H(\vec{g})\right|_{p_{k}}=A^{-1} b$ is computed by fitting a hyperplane through sample $\vec{p}_{k}$ based on its $V$ neighbours $\left\{\vec{p}_{k v}\right\}_{v=1}^{V}$, provided that $A(i, j)=\left(p_{k i}^{(j)}-p_{k}^{(j)}\right)$ and $b(i, 1)=H\left(\vec{p}_{k i}\right)$ for $i=1, . ., V$ and $j=1, \ldots, N$. Once the gradient is estimated, the dynamical behavior around sample $\vec{p}_{k}$ is quantified by comparing the response of $\tilde{H}(\vec{g})$ with the true response $H(\vec{g})$ at the neighboring samples $\vec{p}_{k v}$. A large deviation metric

$$
\bar{E}\left(\vec{p}_{k}\right)=\sum_{v=1}^{V}\left|\tilde{H}\left(\vec{p}_{k v}\right)-H\left(\vec{p}_{k v}\right)\right|
$$

indicates regions where the data is varying more rapidly. To obtain a normalized metric $E\left(\vec{p}_{k}\right) \in[0,1]$, one defines

$$
E\left(\vec{p}_{k}\right)=\frac{\bar{E}\left(\vec{p}_{k}\right)}{\bar{E}\left(\vec{p}_{1}\right)+\ldots+\bar{E}\left(\vec{p}_{K}\right)}
$$

This metric $E\left(\vec{p}_{k}\right)$ quantifies the portion of the dynamic variation in the response that is located near data sample $\vec{p}_{k}$.

\section{Vi. Sequential Data Sample Selection}

The exploration-based metric $V\left(\vec{p}_{k}\right)$ in (1) quantifies data samples according to the size of their corresponding Voronoi cell, while the exploitation-based metric $E\left(\vec{p}_{k}\right)$ in (6) quantifies samples according to the local variation of the response. Both are combined into a global metric that is used for ranking.

$$
G\left(\vec{p}_{k}\right)=\left(1+V\left(\vec{p}_{k}\right)\right)\left(1+E\left(\vec{p}_{k}\right)\right)
$$

Data samples associated with large values of (7) are located in regions which are likely undersampled, whereas the smaller values of (7) correspond to regions that are sampled sufficiently dense. If the data sample with the maximum value of (7) is denoted by $\vec{p}_{m}$, then the algorithm select an additional data sample inside the Voronoi cell $C_{m}$. Its exact location is chosen in such a way that the distance from the neighbours $N\left(\vec{p}_{m}\right)$ is maximized. Once the new data sample is added to $P$, the procedure is repeated until the algorithm is terminated.

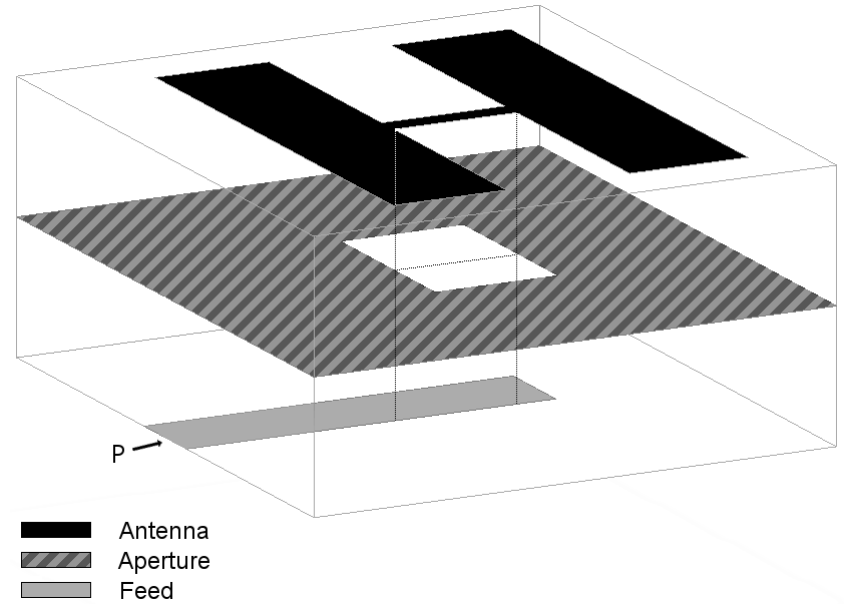

Fig. 1. 3-D view of the microwave H-Antenna
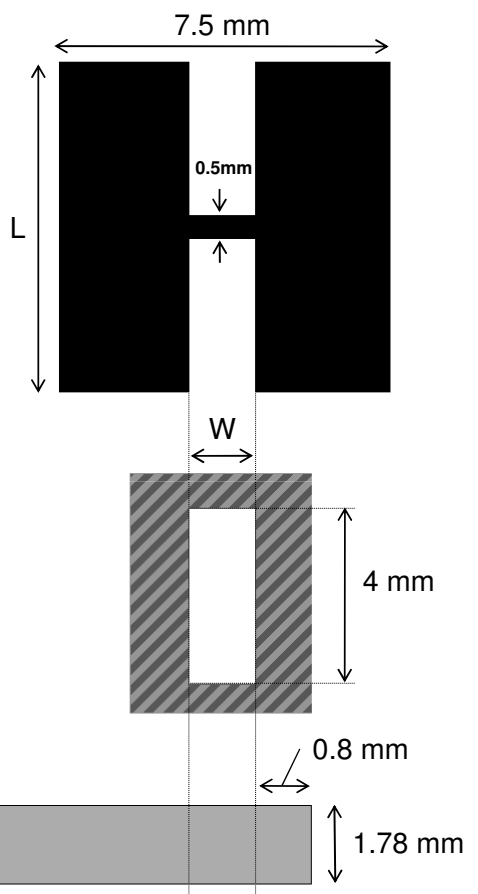

Fig. 2. Top view of the microwave H-Antenna

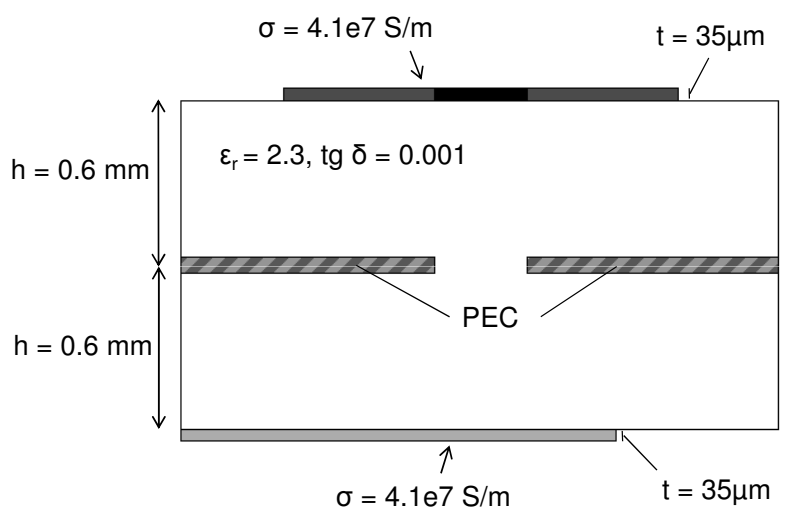

Fig. 3. Cross-section of the microwave H-Antenna 


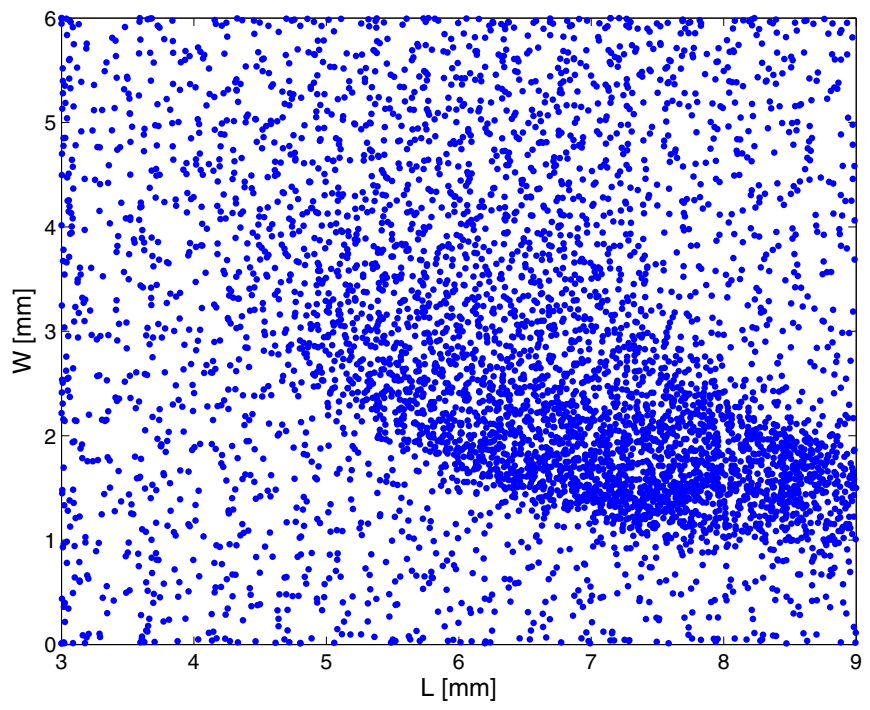

Fig. 4. Data sample distribution $W-L$ space ( $f$ projected)

\section{EXAMPle : MicRowAVE H-ANTENNA}

This example deals with the parametric macromodeling of the reflection coefficient $S_{11}$ of a scalable $\mathrm{H}$-shaped microwave antenna. Fig. 1 shows a 3-D view of the antenna, which consists of three layers: a top layer with the H-shaped antenna, a bottom layer with the feed line and a middle slot layer with a rectangular aperture that realizes the coupling between the feed and the antenna. Fig. 2 shows a top view of the three metal layers along with their respective dimensions. A cross-section of the structure is shown in Fig. 3, depicting the vertical position of the metal layers in the dielectric. The design parameters of the model are the frequency $f$, the length $L$ of the antenna and width $W$ of the aperture. The parameter ranges of interest are set to $f \in[4.5-5.5] \mathrm{GHz}, L \in[3-9]$ $\mathrm{mm}$ and $W \in[0.01-6] \mathrm{mm}$. All data samples are selected by the sequential sampling algorithm, and simulated with the full-wave electromagnetic simulator ADS Momentum [23].

The algorithm starts by simulating a limited set of data samples, and sequentially computes additional data samples that are scattered in the design space. Based on the combined metric function (7), the neighbourhood of each data sample is ranked, and the undersampled regions of the design space are identified. In successive iteration steps, additional data samples are selected until the overall response is well resolved. The distribution of the data samples is shown in Figs. 4-6. (For ease of representation, samples are visualized by projecting the remaining parameter on a 2-dimensional parameter space).

To validate the effectiveness of the sample distribution, the parametrized system response is simulated for a constant value of $L=6 \mathrm{~mm}$ and a varying width $W$ and frequency $f$. In terms of the design space, this corresponds to the horizontal solid line (red) that is shown in Fig. 5. It is seen from this figure that data samples are distributed more densely at frequencies higher than -approximately- $4.8 \mathrm{GHz}$, as marked

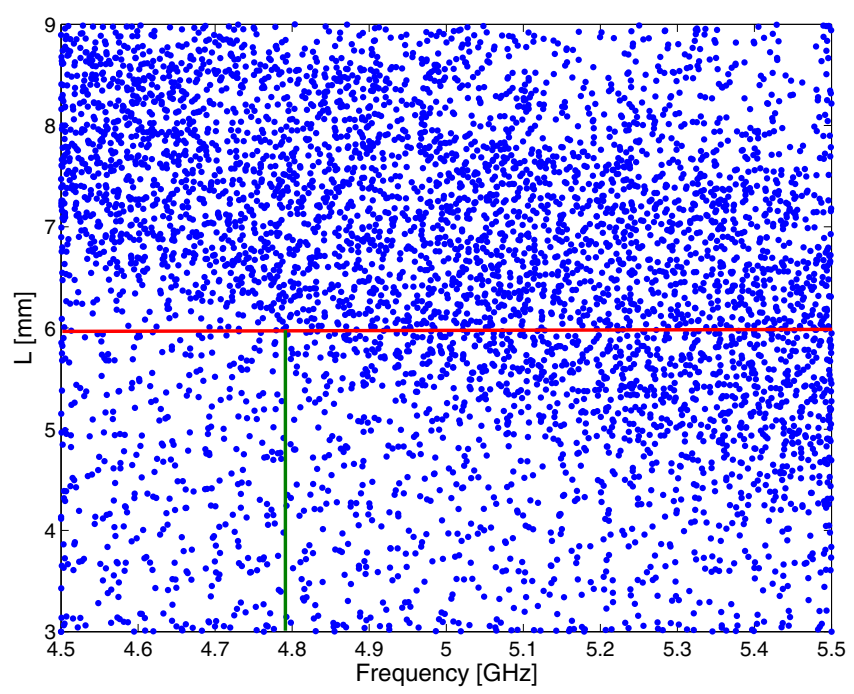

Fig. 5. Data sample distribution $L-f$ space ( $W$ projected)

by the vertical solid line (green). The reason becomes clear when Fig. 7 is considered. The response shows a resonant behavior that changes as a function of $W$ and involves mostly these higher frequencies. This is also outlined by the vertical solid line (green). At the lower frequencies, the response is smooth and shows little variation, which indeed confirms that a sparser sampling is sufficient to capture the dynamics.

As an additional test, the system response is simulated for a constant frequency, e.g. $f=5 \mathrm{GHz}$, and a varying width $W$ and length $L$. This corresponds to the vertical solid line (red) shown in Fig. 6. Here, it is also found that the data samples are distributed more densely if $W$ has a value larger than -approximately- $1 \mathrm{~mm}$, as marked by the horizontal solid line (green). Numerical simulations in Fig. 8 confirm that the response shows a resonant behavior over this part of $W$ 's parameter range. The variation of the response is especially dynamic inbetween $1 \mathrm{~mm}$ and $2 \mathrm{~mm}$, which corresponds to the dense clustering of the data samples observed in Fig. 6.

These results confirm that the dynamical regions of the design space are indeed sampled more densely than other regions where the frequency response shows less variation.

\section{CONCLUSION}

In this paper, an efficient sequential sampling strategy was proposed for accurate modeling of S-parameter based system responses. It automatically selects a set of data samples at scattered locations in the design space, in such a way that the overall dynamics of the response are captured. The method can be linked to any full-wave electromagnetic simulator, and does not require prior knowledge of the device under test. The new approach is more generic than many existing sampling strategies, in a sense that the selection of data samples is completely decoupled from the model type selection (rational functions, neural networks, ...). Application of the technique to an antenna example confirms the effectiveness of the approach. 


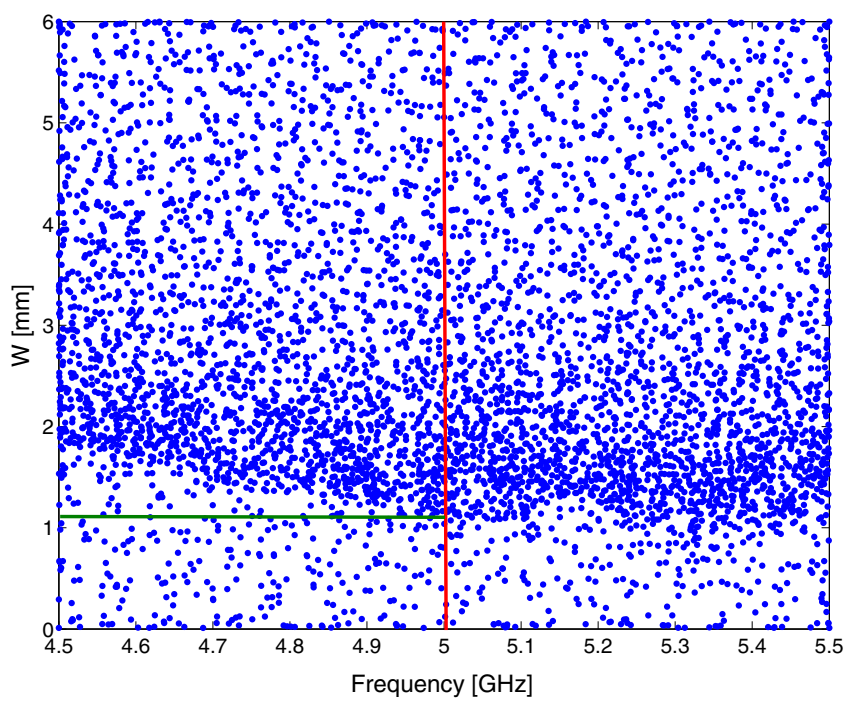

Fig. 6. Data sample distribution $W-f$ space ( $L$ projected)

\section{ACKNOWLEDGMENT}

This work was supported by the Fund for Scientific Research Flanders (FWO-Vlaanderen). Dirk Deschrijver is a post-doctoral research fellow of FWO-Vlaanderen.

\section{REFERENCES}

[1] B. Gustavsen, A. Semlyen, "Rational Approximation of Frequency Domain Responses by Vector Fitting ", IEEE Transactions on Power Delivery, vol. 14, no. 3, pp. 1052-1061, 1999.

[2] D. Deschrijver, T. Dhaene, "A Note on the Multiplicity of Poles in the Vector Fitting Macromodeling Method", IEEE Transactions on Microwave Theory and Techniques, vol. 55, no. 4, pp. 736-741, 2007.

[3] P. Triverio, S. Grivet-Talocia, M. Nakhla, F. Canavero, R. Achar, "Stability, Causality and Passivity in Electrical Interconnect Models, IEEE Transactions on Advanced Packaging, vol. 30, no. 4, pp 795-808, 2007.

[4] W. Hendrickx, D. Deschrijver, T. Dhaene, "Some Remarks on the Vector Fitting iteration", Progress in Industrial Mathematics at ECMI 2004, Mathematics in Industry, vol. 8, Springer, pp. 134-138, 2006.

[5] C. Saunders, J. Hu, C. Christoffersen, M. Steer, "Inverse Singular Value Method for Enforcing Passivity in Reduced-Order Models of Distributed Structures for Transient and Steady-State Simulation", IEEE Trans. on Microwave Theory and Techniques, vol. 59, no. 4, pp. 837-847, 2011.

[6] T. Dhaene, D. Deschrijver, "Generalised Vector Fitting Algorithm for Macromodeling of Passive Electronic Components", Elec. Lett., vol. 41, 6, 299-300, 2005.

[7] G. Song, L. Yu-Shan, Z. Mu-Shui,"An Efficient Algebraic Method for the Passivity Enforcement of Macromodels ", IEEE Trans. on Microwave Theory and Techniques, vol. 58, no. 7, pp. 1830-1839, 2010.

[8] D. Deschrijver, T. Dhaene, "Stability and Passivity Enforcement of Parametric Macromodels in Time and Frequency Domain", IEEE Transactions on Microwave Theory and Techniques, vol. 56, no. 11, pp. 2435-2441, 2008.

[9] E. Laporte, P. Le Tallec, "Numerical Methods in Sensitivity Analysis and Shape Optimization", Birkhauser, Boston, 2003.

[10] D. Gorissen, I. Couckuyt, P. Demeester, T. Dhaene and K. Crombecq, "A Surrogate Modeling and Adaptive Sampling Toolbox for Computer Based Design", Journal of Machine Learning Research, vol. 11, pp. 2051-2055, 2010.

[11] D. Deschrijver, K. Crombecq, H. M. Nguyen, T. Dhaene, "Adaptive Sampling Algorithm for Macromodeling of Parameterized S-Parameter Responses", IEEE Trans. on Microwave Theory and Techniques, vol. 59, no. 1, pp. 39-45, 2011.

[12] K. Crombecq, D. Gorissen, D. Deschrijver, T. Dhaene, "A Novel Hybrid Sequential Design Strategy for Global Surrogate Modelling of Computer Experiments", SIAM Journal on Scientific Computing, vol. 33, no. 4, pp. 1948-1974, 2011.

[13] S. F. Peik, R. R. Mansour and Y. L. Chow, "Multidimensional Cauchy Method and Adaptive Sampling for an Accurate Microwave Circuit Modelling", IEEE Trans. on Microwave Theory and Techniques, vol. 46, pp. 2364-2371, December 1998.

[14] J. De Geest, T. Dhaene, N. Fache and D. De Zutter, "Adaptive CAD-model building Algorithm for General Planar Microwave Structures", IEEE Transaction s on Microwave Theory and Techniques, vol. 47, pp. 1801-1809, September 1999.

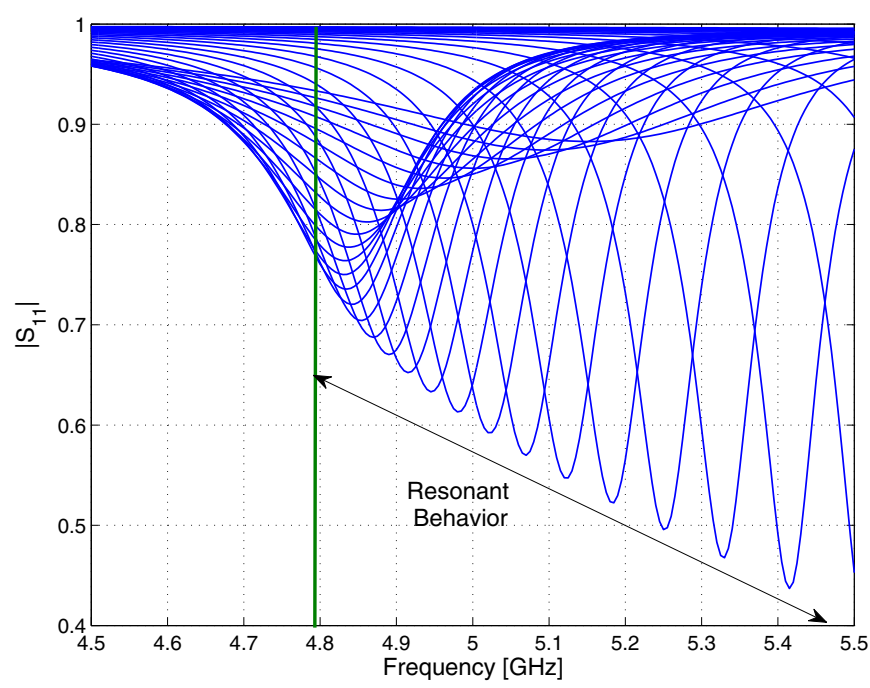

Fig. 7. Magnitude of reflection coefficient $S_{11}$ if $L=6 \mathrm{~mm}$, simulated for several discrete values of $0.01 \mathrm{~mm} \leq W \leq 6 \mathrm{~mm}$

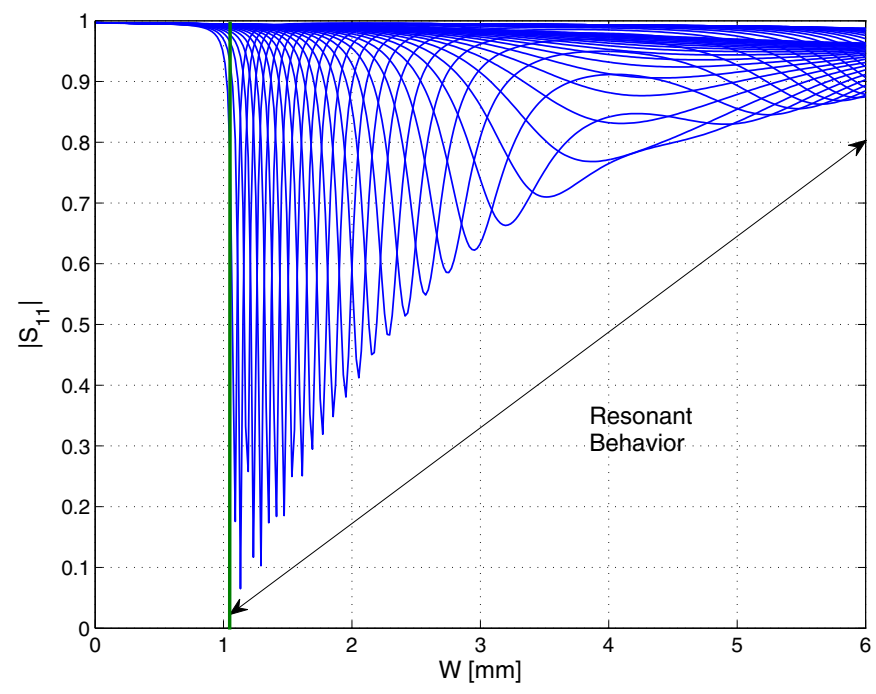

Fig. 8. Magnitude of reflection coefficient $S_{11}$ if $f=5 \mathrm{GHz}$, simulated for several discrete values of $3 \mathrm{~mm} \leq L \leq 9 \mathrm{~mm}$

[15] R. Lehmensiek and P. Meyer, "Creating Accurate Multivariate Rational Interpolation Models for Microwave Circuits by using Efficient Adaptive Sampling to Minimize the Number of Computational Electromagnetic Analyses", IEEE Trans. on Microwave Theory and Techniques, vol. 49, no. 8, pp. 1419, August 2001.

[16] A. Lamecki, P. Kozakowski and M. Mrozowski, "CAD-model Construction Based on Adaptive Radial Basis Functions Interpolation Technique", $15^{\text {th }}$ Int. Conf. on Microwaves, Radar and Wireless Communications, vol. 3, pp. 799-802, May 2004.

[17] J.P.C. Kleijnen and W.C.M. Van Beers, "Application Driven Sequential Designs for Simulation Experiments: Kriging Metamodels", Journal of the Operational Research Society, vol. 55, pp. 876-883, December 2004.

[18] D. C. Montgomery, "Design and Analysis of Experiments", Wiley, 5th edition

[19] A. Forrester, A. Sobester, A. Keane, "Engineering Design via Surrogate Modelling A Practical Guide", Wiley, 2008.

[20] F. Aurenhammer, "Voronoi Diagrams - A Survey of a Fundamental Geometric Data Structure", ACM Computing Surveys, vol. 23, no. 3, pp. 345-405, 1991.

[21] N. Metropolis and S. Ulam, "The Monte Carlo Method", Journal of American Statistical Association, vol. 44, no. 247, pp. 335-341, 1949.

[22] T. Hachisuka, W. Jarosz, R. P. Weistroffer, K. Dale, G. Humphreys, M. Zwicker, H. W. Jensen, "Multidimensional Adaptive Sampling and Reconstruction for Ray Tracing", ACM Transactions on Graphics, vol. 27, no. 3, 10 pages, 2008.

[23] Agilent EEsof COMMS EDA, ADS Momentum Software, Santa Rosa, CA 